\title{
A Single Growth Cone is Capable of Integrating Simultaneously Presented and Functionally Distinct Molecular Cues during Target Recognition
}

\author{
Demian Rose and Akira Chiba \\ Department of Cell and Structural Biology, University of Illinois, Urbana, Illinois 61801
}

A variety of cell recognition pathways affect neuronal target recognition. However, whether such pathways can converge at the level of a single growth cone is not well known. The RP3 motoneuron in Drosophila has previously been shown to respond to the muscle cell surface molecules TOLL and fasciclin III (FAS3), which are normally encountered during RP3 pathfinding in a sequential manner. TOLL and FAS3, putative "negative" and "positive" recognition molecules, respectively, affect RP3 antagonistically. Under normal conditions, TOLL and FAS3 together improve the accuracy of its target recognition. Here, we show that, when presented with concurrent TOLL and FAS3 expression, RP3 responds to both, integrating their effects. This was demonstrated most succinctly by single cell visualization methods. When a balance in relative expression levels between

Neuronal growth cones are capable of responding to their molecular environments. Goodman and Tessier-Lavigne (1996) summarize four functional classes of extrinsic cues that influence growth cone behaviors: diffusible attractive cues, cell-bound attractive cues, diffusible repulsive cues, and cell-bound repulsive cues. Each class is represented by structurally diverse molecules. In the developing brain, individual growth cones encounter many such recognition molecules. However, how each growth cone integrates multiple recognition signals is not well understood.

Recent studies support the idea that a growth cone is a site of molecular integration on which multiple signaling pathways converge. In vitro, the response of a growth cone to specific extrinsic cues depends crucially on its internal cAMP, cGMP, and calcium levels, suggesting that these second messengers may be key components of signal integrations (Loschinger et al., 1997; Ming et al., 1997; Song et al., 1997; Kuhn et al., 1998). In vivo, axons of Caenorhabditis elegans are guided by netrin and TGF- $\beta$, two distinct diffusible cues that may be encountered simultaneously (Colavita et al., 1998). Similarly, synaptic target recognition by many Drosophila motoneurons apparently depends on the balance between "attractive" netrins and "repulsive" semaphorins, both normally derived from overlapping subsets of muscles (Winberg et al., 1998). An emerging idea is that the specific behavior of a

Received Oct. 29, 1998; revised March 10, 1999; accepted March 25, 1999.

This work was supported by grants from National Institutes of Health (NS35049), National Science Foundation (IBN-95-14531), and the Lucille P. Markey Charitable Trust (A.C.). We thank John Cho (University of Illinois) for invaluable help with immunocytochemical data collection, Carl Hashimoto (Yale University) and Corey Goodman (University of California) for reagents, and the current members of the Chiba Lab (University of Illinois) for comments on this manuscript.

Correspondence should be addressed to Demian Rose, B605 Chemical and Life Science Laboratory, 601 South Goodwin Avenue, Urbana, IL 61801.

Copyright (C) 1999 Society for Neuroscience $0270-6474 / 99 / 194899-08 \$ 05.00 / 0$ the two antagonistic cues is achieved, the RP3 growth cone exhibits a phenotype virtually identical to that seen when neither TOLL nor FAS3 is misexpressed. Thus, growth cones are capable of quantitatively evaluating distinct recognition cues and integrating them to attain a net result, in effect responding to the "balance of power" between positive and negative influences. We suggest that the ability to integrate multiple recognition pathways in real-time is one important way in which an individual growth cone interprets and navigates complex molecular environments.

Key words: axon guidance; Drosophila; fasciclin III; growth cone; neuromuscular; neuronal recognition; signal integration; synaptic target recognition; pathfinding; TOLL growth cone results from signal integrations that reflect the particular combination and balance of the molecular cues surrounding it.

In this study, we tested this idea in vivo at the level of a single growth cone as it makes decisions about synaptic targets. We chose to focus on the RP3 motoneuron growth cone in the Drosophila embryonic nervous system (Chiba and Rose, 1998). Synaptic target recognition of this well studied growth cone depends on various muscle-derived cues, including TOLL, fasciclin III (FAS3), capricious, semaphorins, and netrins (Nose et al., 1994; Chiba et al., 1995; Matthes et al., 1995; Kopczynski et al., 1996; Kose et al., 1997; Rose et al., 1997; Shishido et al., 1998). TOLL and FAS3 exert, respectively, negative and positive influence on RP3 synaptic initiation. During normal development, the RP3 growth cone encounters cell-bound TOLL protein in the proximal muscles $(15,16,17$, and 28$)$ before reaching its eventual synaptic target muscles ( 6 and 7 ), which express cell-bound FAS3 (Fig. 1). This sequential encounter with TOLL and FAS3, as well as the response elicited by each, is thought to contribute to the highly accurate synaptic target recognition of the RP3 growth cone. What would be the response of the RP3 growth cone if it simultaneously encountered both TOLL and FAS3, each of which is known to elicit a functionally distinct response from the growth cone?

We show that the RP3 growth cone is capable of integrating the effects of simultaneously presented TOLL, a synapse-inhibiting cue, and FAS3, a synapse-promoting cue. When a balance in the relative expression levels is achieved between these two antagonistic cues at specific recognition sites, RP3 exhibits a phenotype similar to that seen when neither TOLL nor FAS3 is misexpressed. Furthermore, our data suggest that RP3 cannot only respond to TOLL and FAS3 simultaneously but can also evaluate 


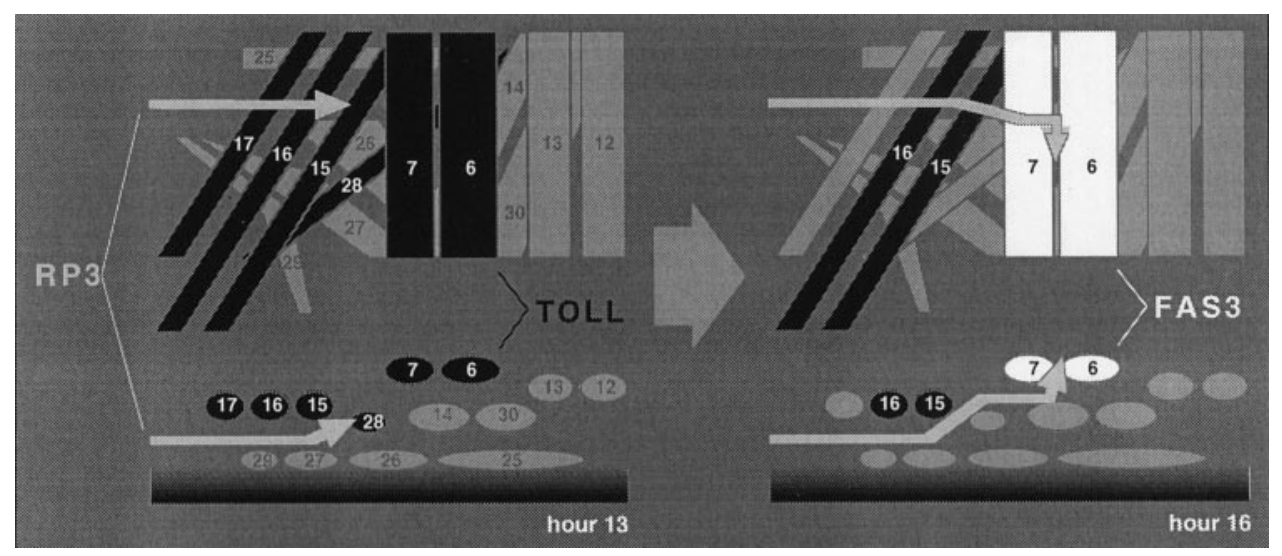

Figure 1. Wild-type presentation of TOLL and FAS3 is sequential. Schematic dorsal (top) and cross-sectional (bottom) views illustrate both TOLL and FAS3 are normally encountered by the RP3 motoneuron growth cone during pathfinding in the ventral musculature (Kose et al., 1997 and Rose et al., 1997, their immunological data). After leaving the CNS at hour 12 of embryogenesis, the RP3 growth cone (gray arrows) contacts a proximal musculature (muscles 6, 7, 15, 16, 17, and 28) enriched with TOLL protein (black). Although TOLL is expressed on muscles 6 and 7 at this time, it is downregulated before the time by which RP3 initiates synaptogenesis (hour 15). FAS3 expression (white) occurs at hours 14-18. FAS3 protein is restricted to the surfaces of muscles 6 and 7, the eventual targets of the RP3 growth cone.

their relative importance. Our results provide evidence for signal integration at the level of an individual growth cone and begin to illuminate the ways by which individual growth cones interpret in vivo microenvironments to recognize their synaptic targets.

\section{MATERIALS AND METHODS}

Drosophila stocks. To misexpress both FAS3 and TOLL proteins in the musculature, previously generated transgenic lines homozygous for the $M h c^{\prime}$-TOLL $L^{90-28}$ transgene (insertion on the second chromosome; Rose et al., 1997) and the $M h c^{\prime}-F A S 3^{27}$ transgene (insertion on the third chromosome; Kose et al., 1997) were crossed to create a double homozygous line. In addition, lines heterozygous for either TOLL, FAS3, or both transgenes were generated. Canton S strain served as a wild type.

Immunocytochemistry. Motoneuron phenotypes were scored by monoclonal antibody (mAb) 1D4 immunocytochemistry (Van Vactor et al., 1993). Anti-TOLL $L_{\mathrm{NT}}$ (from Carl Hashimoto, Yale University, New Haven, CT) and anti-FAS3 (mAb 7G10; from Hybridoma Center, Iowa City, IA) were used to visualize protein localization (Patel et al., 1987; Hashimoto et al., 1988). Embryos were immunoprocessed as whole embryos (Kose et al., 1997). Analysis was based on abdominal (A2-A7) segments of fillet-dissected preparations.

Intracellular dye injection into RP3. RP3 cell bodies were injected with fluorescent dye (Lucifer yellow; Molecular Probes, Eugene, OR) in the embryonic CNS at hours 16-18 as described previously (Rose et al., 1997).

\section{RESULTS}

\section{Muscles develop normally in TOLL/FAS3 co-misexpressor embryos}

To simultaneously present TOLL and FAS3 to motoneuron growth cones, we generated a fly line that is doubly homozygous for $M h c^{\prime}-T O L L$ and $M h c^{\prime}-F A S 3$ transgenes. As demonstrated previously, each of these transgenes misexpresses either wild-type TOLL or FAS3 protein in the entire musculature during the period of motoneuron-muscle interaction (Kose et al., 1997; Rose et al., 1997). We chose to combine these two "misexpressor" transgenes, both of which use the same myotopic promoter $\left(M h c^{\prime}\right)$, creating embryos in which both TOLL and FAS3 proteins will be presented to migrating growth cones in a similar spatiotemporal pattern. The TOLL/FAS3 co-misexpressor line, as well as its parental lines, is viable and can be stably maintained as a homozygote.

Testing with TOLL and FAS3 antibodies indicated that the two proteins are simultaneously expressed at elevated levels in all muscles (Fig. 2). As with other muscle cell surface molecules, both TOLL and FAS3 appear to accumulate at muscle-muscle contact sites (Fig. 2, arrowheads). Misexpression occurs throughout most of the period of motoneuron axon pathfinding and synaptogenesis, i.e., hours 12-20 of embryogenesis. Despite transgenic expression, there is no difference in the number or overall morphology of muscles (Fig. $3 B-D$ ).

Therefore, motoneuron growth cones leaving the CNS will experience both TOLL and FAS3 expression on all muscle surfaces that they encounter. This contrasts the wild-type situation in which growth cones first encounter TOLL-expressing proximal muscles $(15,16,17$, and 28) before reaching FAS3-expressing proximal-medial muscles (6 and 7) a few hours later (Fig. 1). Any motoneuron axon defects that result from co-misexpression of
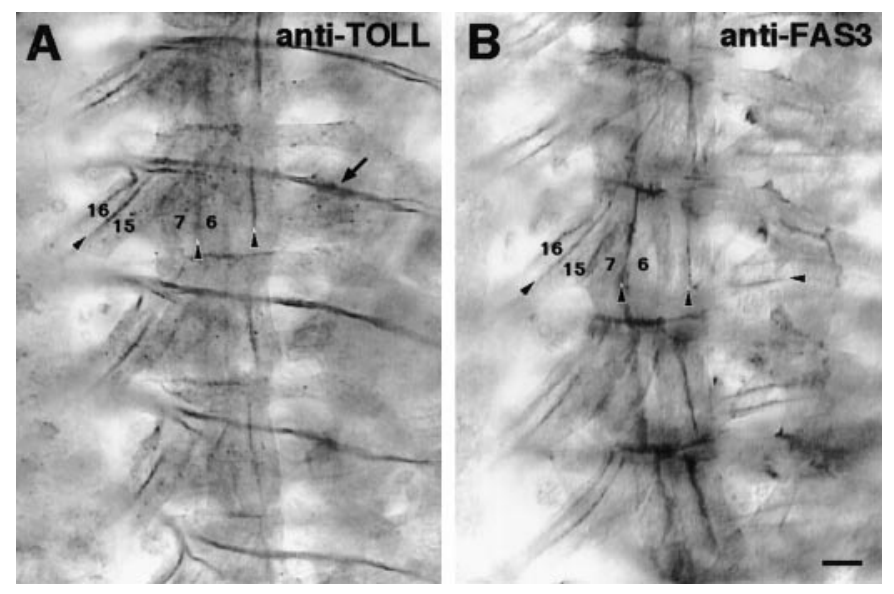

Figure 2. TOLL/FAS3 co-misexpressor embryos express both molecules in muscle tissue. Immunocytochemistry reveals that the $M h c^{\prime}$ promoter drives expression of both TOLL $(A)$ and FAS3 $(B)$ protein on all muscle surfaces in TOLL/FAS3 co-misexpressor embryos at hour 16. Four right-side hemisegments are shown in each panel. Temporal onset of misexpressed TOLL and FAS3 is virtually identical, with high levels detectable at hour 12 and persisting throughout embryonic development. These transmembrane proteins accumulate at muscle-muscle contact sites (arrowheads). Some of the muscles in focus are labeled. Staining of the transverse nerve by anti-TOLL antibody (arrow) is attributable to antibody cross-reactivity and not TOLL expression. Scale bar, $20 \mu \mathrm{m}$. 

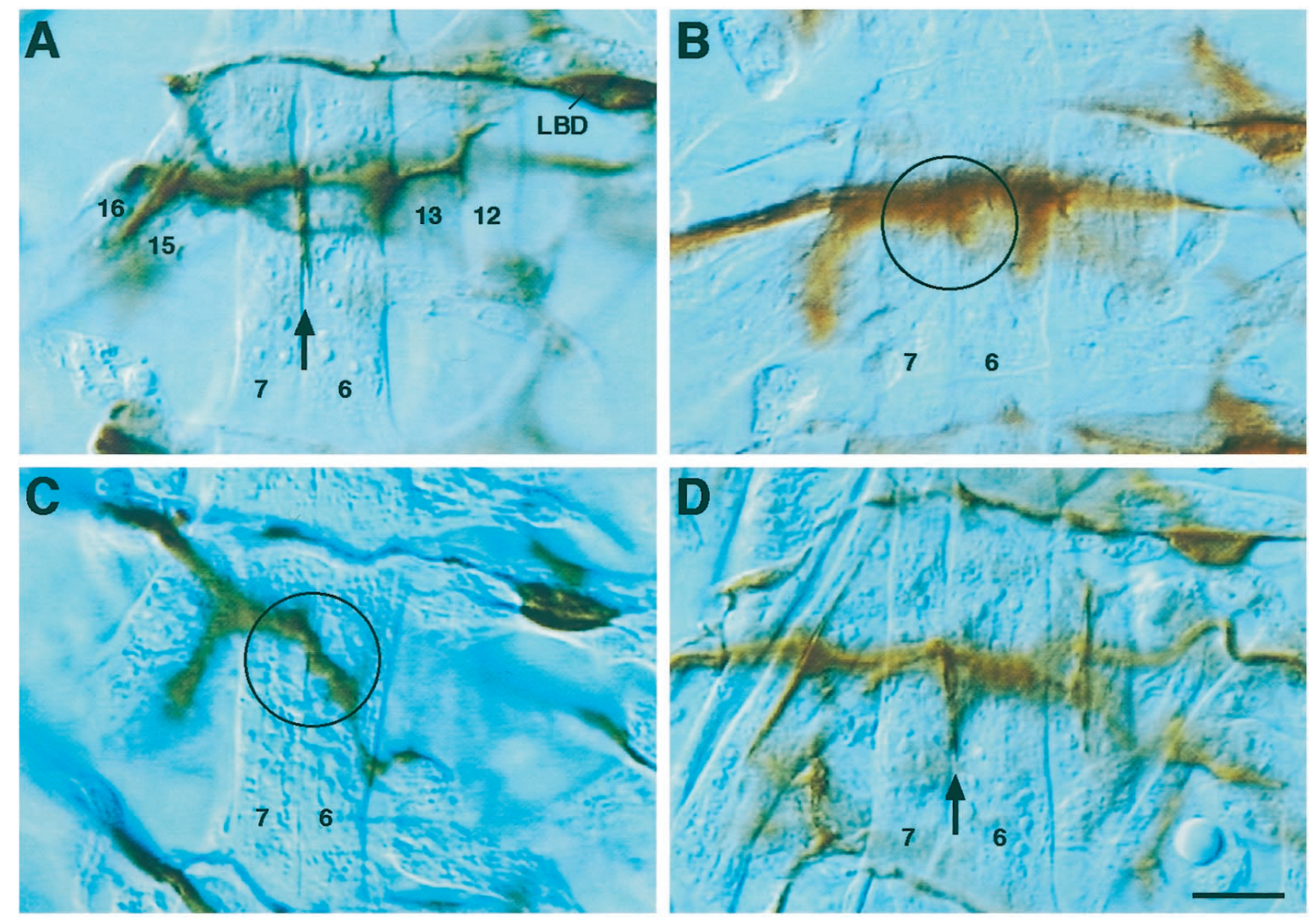

Figure 3. The 6/7 cleft phenotype of TOLL/FAS3 co-misexpressors is similar to wild type and less severe than that of the misexpression of either molecule alone. Hour 18 embryos stained with mAb 1D4 reveal the presence or absence of innervation at the 6/7 muscle cleft. $A$, Wild-type embryo, showing innervation (arrow) at the 6/7 muscle cleft. B, TOLL misexpressor, showing a lack of 6/7 innervation (circle). C, FAS3 misexpressor, showing a lack of 6/7 innervation (circle). Axons of unidentified motoneurons external to the cleft are out of focus. $D$, TOLL/FAS3 co-misexpressor, showing innervation virtually identical to wild type (arrow). All photos were taken at the focal plane of the cleft. Lateral bidendritic $(L B D)$ cell is labeled for positional clarity. Scale bar, $10 \mu \mathrm{m}$. See Table 1 for data summary.

TOLL and FAS3 may be interpreted as a likely direct result of motoneuron growth cones encountering TOLL and FAS3 proteins outside of their normal context.

\section{Nervous system develops normally in TOLL/FAS3 co-misexpressor embryos}

We first examined the overall development of the nervous system in TOLL/FAS3 co-misexpressor embryos. Monoclonal antibody 1D4, which labels all motoneuron axons (Van Vactor et al., 1993), revealed normal overall development of the CNS and PNS. In each hemisegment of the peripheral nervous system, all five major nerve branches [intersegmental nerve, segmental nerve a ( $\mathrm{SNa})$, $\mathrm{SNb}, \mathrm{SNc}$, and $\mathrm{SNd}$ ] extend outside the CNS into characteristic muscle regions. Consistent with previous reports (Chiba et al., 1995; Kose et al., 1997; Rose et al., 1997), the two parental lines (Mhc'-TOLL and $M h c^{\prime}$-FAS3 homozygous lines) also show virtually wild-type-like overall development of their nervous systems.

Previous studies using the $M h c^{\prime}-T O L L$ and $M h c^{\prime}-F A S 3$ lines revealed that subsets of motoneuron growth cones are often misguided when encountering ectopic TOLL or FAS3 expression in the musculature (Chiba et al., 1995; Kose et al., 1997; Rose et al., 1997). In particular, RP3, which normally innervates the cleft between muscles 6 and 7 (Keshishian and Chiba, 1993), fails to reach the 6/7 cleft at a frequency $>50 \%$ (Chiba et al., 1995; Rose et al., 1997). We confirmed these observations in a new data set analyzed at hours 16-18 of embryogenesis (Fig. 3B, C, Table 1).

In contrast to the situations in these parental lines, the $6 / 7 \mathrm{cleft}$ in TOLL/FAS3 co-misexpressor embryos remains innervated at a high frequency when examined with immunocytochemistry (Fig. 3D, Table 1). Both the rate (90 vs 94\%) and the morphological appearance of innervation at the 6/7 cleft (Fig. 3, compare $A, D)$ are very similar to those of wild type.

This apparent return to a wild-type innervation pattern at the 6/7 cleft could be attributable to one of at least two separate situations. Either RP3 or MN6/7b, another motoneuron which innervates the 6/7 cleft (Keshishian and Chiba, 1993), could once again be attracted to the cleft when the levels of TOLL and FAS3 expression there are equalized. Alternatively, the presence of $\mathrm{mAb} 1 \mathrm{D} 4$ staining at the cleft could indicate that a motoneuron other than RP3 or MN6/7b is innervating the cleft ectopically. The latter would imply that co-misexpression of TOLL and FAS3 permits ectopic innervation at the 6/7 cleft more readily than misexpression of either cue alone. The former would suggest that the 6/7 cleft becomes "normalized" when both TOLL and FAS3 are co-misexpressed in the musculature.

\section{The RP3 growth cone can respond simultaneously to TOLL and FAS3}

To distinguish between these possibilities, we visualized the RP3 growth cone directly. We injected intracellular fluorescent dye into RP3 cell bodies in TOLL/FAS3 co-misexpressor embryos at hours 16-18 (see Materials and Methods). By this time, RP3 pathfinding is normally completed (Rose et al., 1997). Such analysis circumvents the ambiguity inherent in mAb 1D4 immunolabeling, because we are able to observe the RP3 phenotype independent of all other motoneurons. 


\begin{tabular}{|c|c|c|c|c|c|c|c|}
\hline \multirow[b]{2}{*}{ Genotype } & \multicolumn{4}{|c|}{ RP3 growth cone (Lucifer yellow) } & \multicolumn{3}{|c|}{ Innervation at $6 / 7$ (mAb 1D4) } \\
\hline & $n$ & target $6 / 7$ & stall & mistarget & $n$ & present & absent \\
\hline \multicolumn{8}{|l|}{ Wild type } \\
\hline$+/+;+/+$ & 11 & $100 \%$ & $0 \%$ & $0 \%$ & 100 & $94 \%$ & $6 \%$ \\
\hline \multicolumn{8}{|c|}{ Group A (steep dosage disparity) } \\
\hline TOLL/TOLL; +/+ & $14^{a}$ & $14 \%$ & $86 \%$ & $0 \%$ & 121 & $46 \%$ & $54 \%$ \\
\hline$+/+; F A S 3 / F A S 3$ & $11^{b}$ & $18 \%$ & $18 \%$ & $64 \%$ & 120 & $45 \%$ & $55 \%$ \\
\hline \multicolumn{8}{|c|}{ Group B (intermediate dosage parity) } \\
\hline TOLL/TOLL; FAS3/+ & 0 & $\mathrm{n} / \mathrm{a}$ & $\mathrm{n} / \mathrm{a}$ & $\mathrm{n} / \mathrm{a}$ & 97 & $67 \%$ & $33 \%$ \\
\hline$T O L L /+; F A S 3 / F A S 3$ & 0 & $\mathrm{n} / \mathrm{a}$ & $\mathrm{n} / \mathrm{a}$ & $\mathrm{n} / \mathrm{a}$ & 102 & $66 \%$ & $34 \%$ \\
\hline \multicolumn{8}{|l|}{ Group C (dosage parity) } \\
\hline TOLL/+; FAS3/+ & 0 & $\mathrm{n} / \mathrm{a}$ & $\mathrm{n} / \mathrm{a}$ & $\mathrm{n} / \mathrm{a}$ & 108 & $94 \%$ & $6 \%$ \\
\hline TOLL/TOLL; FAS3/FAS3 & 13 & $84 \%$ & $8 \%$ & $8 \%$ & 126 & $90 \%$ & $10 \%$ \\
\hline
\end{tabular}

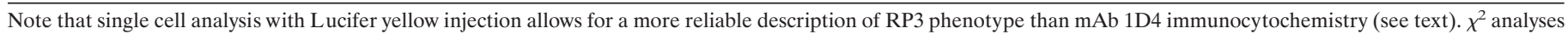

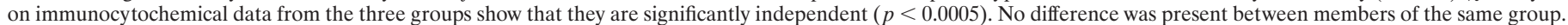

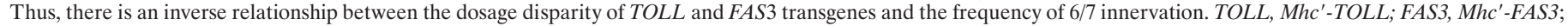
$\mathrm{n} / \mathrm{a}$, data not available.

${ }^{a}$ From Rose et al., 1997.

${ }^{b}$ From Kose et al., 1997.

We noted that, in all cases $(n=13)$, the RP3 axon extends out of the CNS normally. It first crosses the dorsal midline of the CNS and then exits via the intersegmental nerve one segment posterior to its origin. This supports the notion that comisexpression of TOLL and FAS3 in the embryonic musculature does not affect RP3 axonal pathfinding within the CNS.

Once outside the CNS and when encountering concurrent misexpression of TOLL and FAS3, the most common decision of the RP3 growth cone is to navigate through the normal peripheral pathway and to innervate the $6 / 7 \mathrm{cleft}$, its wild-type site of innervation (Fig. $4 D_{1}$, Table 1). As in other studies, we define synaptogenesis initiation based on characteristic morphological changes in the growth cone: filopodial retraction and the lining of a target muscle edge (Sink and Whitington, 1991; Chiba et al., 1993; Matthes et al., 1995; Kose et al., 1997; Rose et al., 1997). In wild type, RP3 initiates synaptogenesis at the muscle 6/7 cleft in $100 \%$ of cases (Fig. 4A, Table 1), consistent with previous studies (Sink and Whitington, 1991; Chiba et al., 1993; Matthes et al., 1995; Kose et al., 1997; Rose et al., 1997). When TOLL alone is misexpressed, the RP3 innervation rate drops to $14 \%$ (Table 1) (Rose et al., 1997). In most cases, the growth cone stalls just beneath (external to) the cleft and fails to initiate synaptogenesis, at least during hours 16-18 (Fig. 4B) (Rose et al., 1997). However, when FAS3 is co-misexpressed with TOLL, the RP3 innervation rate climbs to $84 \%$, approaching the wild-type level (Table $1)$. In the remaining small percentages, the RP3 growth cone either stalls beneath the $6 / 7$ cleft, resembling the most common phenotype with TOLL misexpression alone (Fig. $4 D_{2}$ ), or mistargets muscles 15 and 16, simulating the conditions with FAS3 misexpression alone (Fig. $4 D_{3}$ ). Overall, whereas TOLL is capable of preventing RP3 innervation of its normal target site, comisexpression of FAS3 and TOLL leads to a phenotype that is similar to wild type (Fig. 4D). We interpret these observations as RP3 growth cone integration of two antagonistic signals during its target recognition.

Additional support for signal integration by the RP3 growth cone comes from its implicit behavior at the proximal muscles 15 and 16 . The RP3 growth cone normally avoids synaptogenesis at these muscles. However, these muscles can be converted to ac- ceptable synaptic targets for this growth cone if they ectopically express FAS3 protein (Fig. 4C) (Kose et al., 1997). When muscles bear ectopic TOLL and FAS3 concurrently, the RP3 growth cone does not initiate synaptogenesis at muscles 15 and 16. Instead, when examined at hours $16-18$, the growth cone in the majority of the cases has extended past this site and innervated the 6/7 cleft, just as in wild type (Fig. 4, compare $A, D_{1}$ ). We suggest that the behavior of the RP3 growth cone in TOLL/FAS3 comisexpressors is best described by a growth cone that is capable of actively interpreting and integrating two distinct signaling pathways that are mutually negating, as well as responding to the other cues present.

\section{RP3 responds to relative levels of TOLL and FAS3}

Does the integration of TOLL and FAS3 occur in a summative manner? Would varying the relative levels of each vary the severity of the phenotype of RP3? Because this is a statistical question, we chose immunocytochemistry, with which large sample sizes can be attained, as the means to measure differences in innervation rate at the $6 / 7$ cleft. Based on our intracellular dye injections, we felt confident that nerve staining at the $6 / 7 \mathrm{cleft}$ indicated that RP3 had innervated there.

We generated several additional fly lines, which carry varied dosages of TOLL and FAS3 misexpressor transgenes, and divided the genotypes into three groups based on the degree of dosage disparity (Table 1). Group A defines the case in which the dosage difference between TOLL and FAS3 transgenes is steepest, with one transgene being homozygous (two copies), and its antagonist is not misexpressed at all (no copies). Group B describes an intermediate case with less of a dosage disparity, i.e., homozygous misexpression (two copies) of one transgene and heterozygous misexpression (one copy) of the other. Group $\mathrm{C}$ is the case in which both TOLL and FAS3 are misexpressed at equal genetic dosage levels, being either doubly homozygous (two copies each) or doubly heterozygous (one copy each). If RP3 (and MN6/7b) growth cones are capable of quantitatively evaluating the relative dosages of these two antagonistic recognition cues, we would expect to see the most severe phenotypic deviations from the 

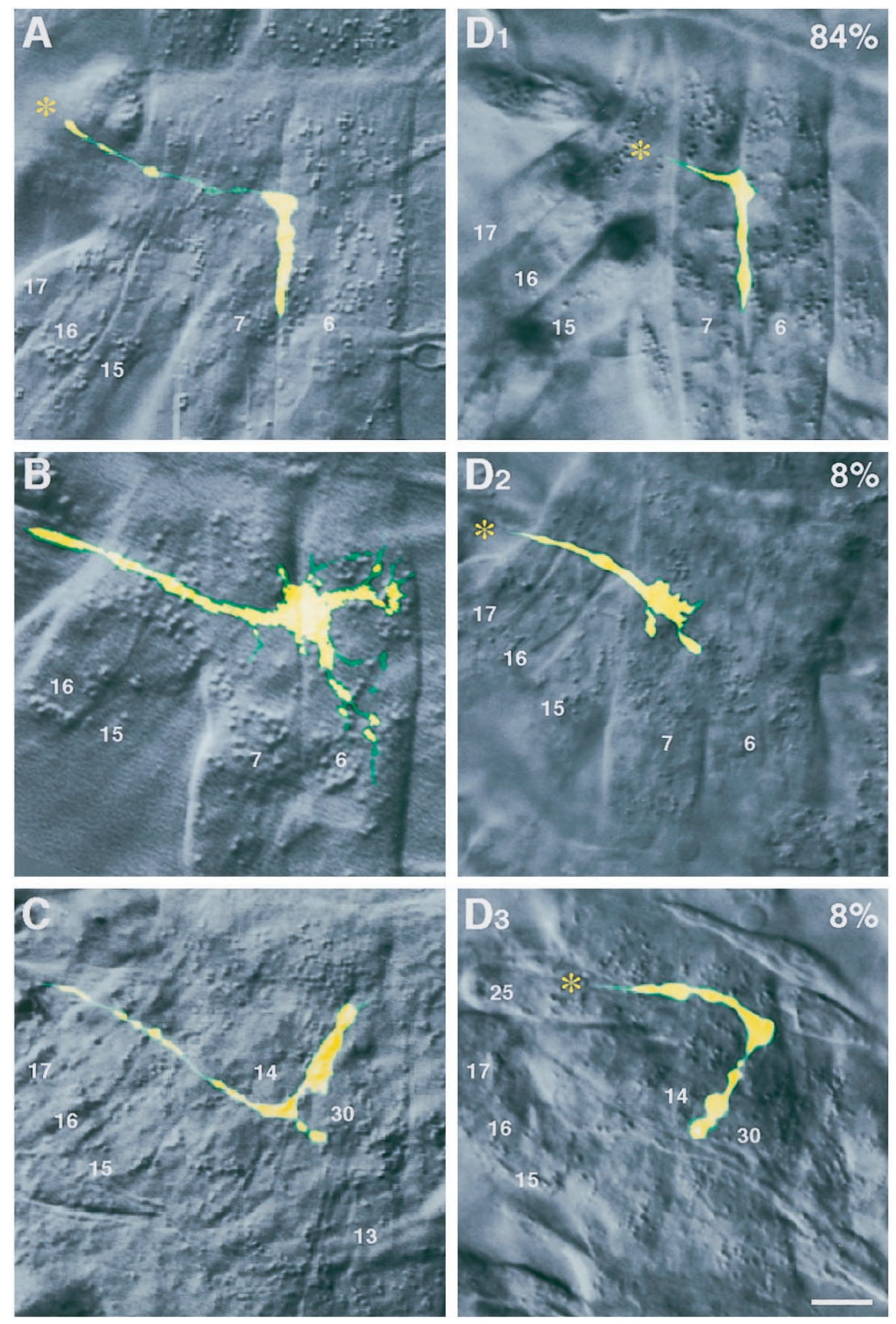

Figure 4. Dye injection reveals the fate of the RP3 growth cone in TOLL/FAS3 co-misexpressors. Fluorescent images of dye-filled RP3 growth cones in hours 16-18 right abdominal hemisegments with corresponding DIC images of the surrounding musculature. $A$, Wildtype embryo, showing innervation of the RP3 growth cone at the 6/7 cleft. $B$, TOLL misexpressor, showing stalling of the RP3 growth cone just underneath (external to) the 6/7 cleft (Rose et al., 1997). Focal plane of DIC image is shown at level of the cleft for clarity. $C$, FAS3 misexpressor, showing misinnervation at the $14 / 30$ muscle cleft, which lies external to muscles 6 and 7 (from Kose et al., 1997). D, TOLL/FAS3 co-misexpressor embryos, showing infrequent RP3 phenotypes that are similar to TOLL or FAS3 misexpressors $(D 2, D 3)$, as well as the most common phenotype, innervation of the $6 / 7 \mathrm{cleft}$ (D1). Asterisks indicate the direction from which the RP3 growth cone originates. Scale bar, $5 \mu \mathrm{m}$. See Table 1 for data summary. wild-type situation in group A (the steepest dosage disparity) and the least severe phenotypes in group C (dosage parity).

The results support this hypothesis. At the 6/7 muscle cleft, the normal site of RP3 and MN6/7b innervation, the three genotypic groups exhibit increasingly less severe phenotypes (Fig. 5, Table $1)$. In group $\mathrm{A}$, we see the most radical departure from the wild-type phenotype, with only $45-46 \%$ of the cases showing innervation at the $6 / 7$ cleft. The "intermediate" group B genotype produces an intermediate phenotype with innervation rates of $66-67 \%$. These rates are less than wild type but more than those of group A. In group C, embryos carrying TOLL and FAS3 transgenes in a 1:1 or 2:2 ratio show a phenotype indistinguishable from wild-type embryos. The rates of $6 / 7$ cleft innervation are $90-94 \%$ compared with $94 \%$ in wild type. Relatively large sample sizes of these immunocytochemical data allowed us to test the statistical significance of differential rates of innervation at the $6 / 7$ cleft. Clearly, there is an inverse relationship between the level of TOLL/FAS3 dosage disparity and the frequency of $6 / 7$ innervation (Fig. 5). It is particularly interesting to note that, although embryos expressing both transgenes homozygously are the furthest from the wild-type situation in terms of molecular expression, they exhibit a phenotype indistinguishable from the wild-type phenotype.

These results suggest a TOLL/FAS3 balancing effect. The two molecules act antagonistically in an integral manner with respect to the RP3 growth cone. Their net effects depend on the relative quantities of each protein expressed on the muscle surface rather than its absolute presence or absence.

\section{DISCUSSION}

This study examined the ability of an individual growth cone to weigh the quantitative contributions of two simultaneously presented, functionally distinct cues and arrive at a decision that reflects their relative expression levels. We showed in vivo that the RP3 growth cone is competent to respond to concurrently misexpressed TOLL and FAS3, two structurally and physiologically 


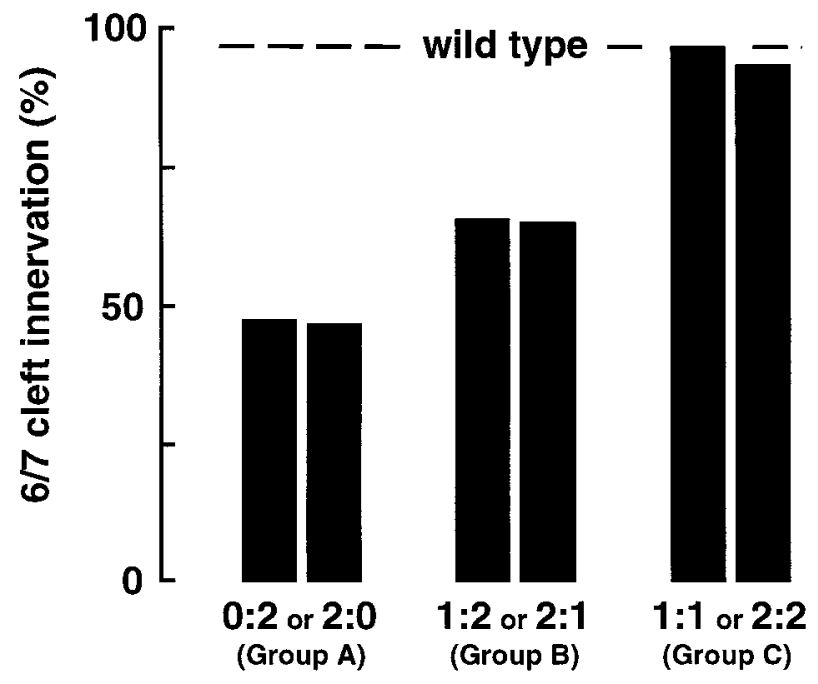

TOLL:FAS3 copy number

Figure 5. The frequency of $6 / 7$ cleft innervation is inversely correlated to TOLL/FAS3 dosage disparity. TOLL:FAS3 dosage variants are divided into three distinct statistical groups based on the relative numbers of $M h c^{\prime}-T O L L$ and $M h c^{\prime}-F A S 3$ transgenes present. In group A, where the levels of TOLL and FAS3 are most disparate (2 copies of one transgene and 0 of the other), innervation at the 6/7 cleft, as scored by immunocytochemistry, is the most infrequent. In group B, as TOLL and FAS3 levels become less disparate ( 2 copies of one transgene and 1 copy of the other), the 6/7 innervation rate is increased. In group C, where the levels of TOLL and FAS3 are equal, the innervation rate at the $6 / 7 \mathrm{cleft}$ is statistically indistinguishable from wild type. See Table 1 for data summary.

distinct molecules normally expressed by the targets of the growth cone and surrounding cells. Furthermore, we presented evidence that the growth cone is capable of evaluating the relative contributions of each molecule and responding appropriately. Our results support the general idea that signal integration at individual growth cones is an important mechanism by which neural networks are established.

\section{Single cell analysis}

Early in this study, it became clear to us that immunocytochemical analyses of motoneuron axon phenotypes do not provide the resolution required to make specific claims regarding RP3 growth cone innervation. With mAb 1D4, which stains all motoneurons, it is possible to score how often RP3 and MN6/7b (Keshishian and Chiba, 1993), the two motoneurons that innervate the cleft between muscles 6 and 7, fail to reach the 6/7 cleft (Fig. 3B,C). However, it is impossible to reliably identify the RP3 growth cone amid the other immunopositive neurons. The absence of immunostaining at the $6 / 7$ cleft gives only a measure of phenotype severity. It cannot distinguish between an RP3 growth cone stalling before reaching the cleft or innervating an alternative target. Additionally, the presence of staining at the cleft does not always mean that RP3 is present. MN6/7b or other ectopic motoneurons may be occupying the innervation site. Therefore, we adopted the single cell approach and conclusively defined the path of RP3 in embryos misexpressing TOLL and FAS3 proteins (Fig. 4D). The primarily wild-type phenotype of co-misexpressor embryos lends strong evidence to the idea that RP3 integrates TOLL and FAS3 signals in real-time.

\section{Signal integration at a single growth cone}

We chose to use the $M h c^{\prime}$ promoter (Wassenberg et al., 1987) as our means to create a controlled environment in which RP3 would see TOLL and FAS3 presented in a similar spatial and temporal context. The transgenic lines we used couple the promoter and cDNA directly. This facilitates a more controlled expression level compared with a heterologous transactivation (GAL4/UAS) system (Brand et al., 1994).

Three lines of evidence support our conclusion that a single growth cone is capable of integrating concurrently presented signals (Table 1). First, although RP3 can be induced to mistarget the $15 / 16$ muscle cleft and other proximal muscles in embryos misexpressing FAS3 alone, the frequency of such misinnervation drops sharply from 59 to $8 \%$ when TOLL is co-misexpressed along with FAS3. Second, although TOLL misexpression is capable of stalling the RP3 growth cone just beneath its normal target site, the frequency of this phenotype drops drastically from 86 to $8 \%$ when FAS3 is co-misexpressed along with TOLL. Third, when the relative genetic dosages of TOLL and FAS3 are varied, we see phenotypes that strongly correlate to the levels of TOLL/FAS3 parity that exist (Fig. 5). These observations support the conclusion that the RP3 growth cone is integrating functionally disparate molecular information in a combinatorial manner.

How universal is this ability to integrate antagonistic cues among growth cones? Winberg et al. (1998) have used immunocytochemical approaches to assess the effect of simultaneously presenting Drosophila motoneuron growth cones with semaphorin II and netrins. Their data are generally consistent with the interpretation that growth cones can simultaneously interpret semaphorin II, a repulsive cue, and netrins, attractive cues. A recent study of the $C$. elegans unc genes, which encode various recognition molecules, also suggests that a combination of attractive and repulsive cues may be ultimately responsible for the dorsoventral polarity information that guides certain subsets of neurons (Colavita et al., 1998). Therefore, signal integration is likely a widely shared feature of neuronal growth cones.

\section{The balance between positive and negative signals during synaptic target recognition}

Our dosage analyses suggest that it is the comparative level of each molecule to which RP3 responds in a summative manner rather than its absolute presence or absence. But where does such
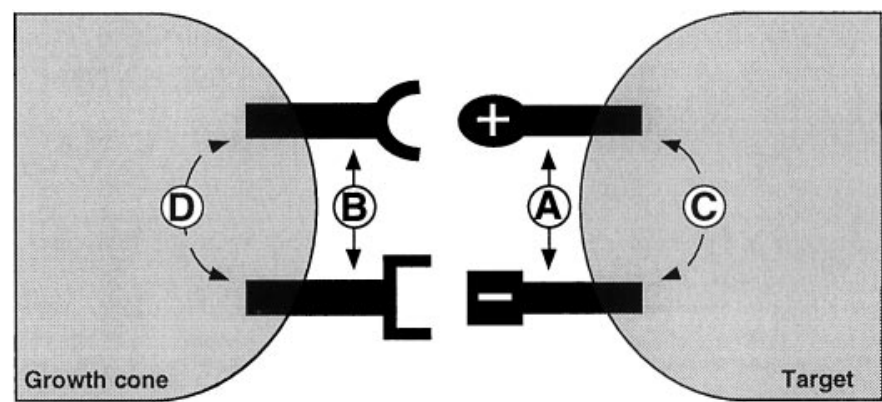

Figure 6. Single growth cone model for antagonistic signal integration. $A-D$, Signal integration of positive and negative cues may occur at any of several different sites. It is possible that cytoplasmic signaling mediated by cell surface molecules on either the target $(C)$ or growth cone $(D)$ side leads to a summative integration of the positive and negative pathways. Alternatively, it is also possible that signal integration occurs on the surface of either the postsynaptic $(A)$ or presynaptic $(B)$ side without requiring intracellular signaling pathways. 
signal convergence occur? In theory, there are at least four possible sites for signal integration of functionally disparate cues at growth cone-target interactions. The first possible site is at the muscle surface, where cell surface molecules interact with each other either directly or indirectly (Fig. 6A). The second possible site where such cell surface signal integration occurs is at the growth cone surface (Fig. 6B). The third and fourth possibilities predict that signal integration occurs beneath the cell surface and is mediated though intracellular signaling pathways at either the postsynaptic (Fig. 6C) or the presynaptic (Fig. 6D) side.

Experiments looking at phosphotyrosine kinases and phosphatases, as well as cAMP and cGMP pathways, suggest that intracellular signaling is a crucial regulator of neural development (Ming et al., 1997; Song et al., 1997; Van Vactor, 1998). It is possible to use essentially the same molecular and cellular techniques described herein to design experiments that will study the cytoplasmic signaling pathways that are required for TOLL/ FAS3 signal integration. For example, by misexpressing constructs of either molecule that lack a cytoplasmic signaling domain, one may be able to demonstrate whether the effects of TOLL and/or FAS3 require signaling within the muscle cytoplasm (Fig. 6C).

Recent electron microscopy work suggests that the effects of TOLL and FAS3 ultimately converge on a common cellular mechanism (E. Suzuki, D. Rose, and A. Chiba, unpublished observations). At the ultrastructural level, TOLL is capable of preventing tight membrane apposition between RP3 and its normal target site. Such selective adhesion is abundantly seen between motoneurons and their normal targets in wild-type embryos. Conversely, FAS3 is able to induce ectopic membrane associations between RP3 and muscles other than its normal target. These observations are consistent with the conclusion that TOLL and FAS3 signaling pathways converge at some point to affect the development of tight membrane contacts between potential synaptic partners. The biochemical bases for such molecular integrations, however, require future investigations.

\section{The "molecular gestalt"}

Molecular signal integration is implicated as a major principle of cell-cell interactions in developing multicellular organisms. For example, the ability of neural progenitors to integrate disparate incoming signals and arrive at a net fate decision is crucial for early brain development (Krumlauf, 1994; Salser and Kenyon, 1994; Tanabe and Jessell, 1996). Similarly, qualitative differences in cell migration appear to be derived from the net quantitative impact of the array of surrounding molecular components (Lauffenburger and Horwitz, 1996). It is conceivable that signal processing required for the establishment of a neural network also heavily relies on the same general principle of signal integration.

The observed responses of the RP3 growth cone to ectopically placed TOLL and FAS3 are consistent with the view that an individual growth cone is an "opportunist" (Chiba and Rose, 1998). In this view, the growth cone is able to respond to many different cues during its normal development, even when presented outside of their normal contexts. Experiments with netrins, semaphorins, connectin, capricious, fasciclin II, and others also suggest that the RP3 growth cone acts in an opportunistic manner (Matthes et al., 1995; Nose et al., 1997; Davis and Goodman, 1998; Shishido et al., 1998; Winberg et al., 1998).

It appears that the local, expedient events of growth cone synaptic target recognition are subject to the same rules that govern cell fate decisions in the early CNS (Tanabe and Jessell, 1996). It seems not to be any one molecule, or even a small group of convergent molecules, to which individual cells are geared to respond. Rather, it is the tightly regulated microenvironment of cues, the molecular gestalt, that is responsible for motoneuron pathfinding. It is ultimately at the level of the growth cone itself where synaptic targeting decisions are made, and it is here that a major integration of the external molecular world must occur. Thus, dynamic integration of external information occurs both at the level of gene transcription of totipotent progenitor cells, as well as during the quickly executed morphological changes seen in synaptic target recognition.

In conclusion, we propose that signal integration is one major principle by which neural networks are formed. Our data suggest that this principle extends to the level of individual growth cones.

\section{REFERENCES}

Brand A, Manoukian AS, Perrimon N (1994) Ectopic expression in Drosophila (Goldstein LSB, Fyrberg EA, eds), pp 683-696. San Diego: Academic.

Chiba A, Rose D (1998) "Painting" the target: how local molecular cues define synaptic relationships. BioEssays 20:941-948.

Chiba A, Hing H, Cash S, Keshishian H (1993) Growth cone choices of Drosophila motoneurons in response to muscle fiber mismatch. J Neurosci 13:714-732.

Chiba A, Snow P, Keshishian H, Hotta Y (1995) Fasciclin III as a synaptic target recognition molecule in Drosophila. Nature 374:166-168.

Colavita A, Krishna S, Zheng H, Padgett RW, Culotti JG (1998) Pioneer axon guidance by UNC-129, a $C$. elegans TGF- $\beta$. Science 281:706-709.

Davis GW, Goodman CS (1998) Synapse-specific control of synaptic efficacy at the terminals of a single neuron. Nature 392:82-86.

Hashimoto C, Hudson KL, Anderson KV (1988) The Toll gene of Drosophila, required for dorsal-ventral embryonic polarity, appears to encode a transmembrane protein. Cell 52:269-279.

Keshishian H, Chiba A (1993) Neuromuscular development in Drosophila: insights from single neurons and single genes. Trends Neurosci 16:278-283.

Kopczynski CC, Davis GW, Goodman CS (1996) A neural tetraspanin, encoded by late bloomer, that facilitates synapse formation. Science 271:1867-1870.

Kose H, Rose D, Zhu X, Chiba A (1997) Homophilic synaptic target recognition mediated by immunoglobulin-like cell adhesion molecule fasciclin III. Development 124:4143-4152.

Krumlauf R (1994) Hox genes in vertebrate development. Cell 78:191-201.

Kuhn TB, Williams CV, Dou P, Kater SB (1998) Laminin directs growth cone navigation via two temporally and functionally distinct calcium signals. J Neurosci 18:184-194.

Lauffenburger DA, Horwitz AF (1996) Cell migration: a physically integrated molecular process. Cell 84:359-369.

Loschinger J, Bandtlow CE, Jung J, Klostermann S, Schwab ME, Bonhoeffer F, Kater SB (1997) Retinal axon growth cone responses to different environmental cues are mediated by different secondmessenger systems. J Neurobiol 33:825-834.

Matthes DJ, Sink H, Kolodkin AL, Goodman CS (1995) Semaphorin II can function as a selective inhibitor of specific synaptic arborizations. Cell 81:631-639.

Ming G, Song H, Berninger B, Holt CE, Tessier-Lavigne M, Poo M (1997) cAMP-dependent growth cone guidance by netrin-1. Neuron 19:1225-1235.

Nose A, Takeichi M, Goodman CS (1994) Ectopic expression of connectin reveals a repulsive function during growth cone guidance and synapse formation. Neuron 13:525-539. 
Nose A, Umeda T, Takeichi M (1997) Neuromuscular target recognition by a homophilic interaction of connectin cell adhesion molecules in Drosophila. Development 124:1433-1441.

Patel NH, Snow PM, Goodman CS (1987) Characterization and cloning of fasciclin III: a glycoprotein expressed on a subset of neurons and axon pathways in Drosophila. Cell 48:975-988.

Rose D, Zhu X, Kose H, Hoang B, Cho J, Chiba A (1997) Toll, a muscle cell surface molecule, locally inhibits synaptic initiation of the RP3 motoneuron growth cone in Drosophila. Development 124:1561-1571.

Salser SJ, Kenyon C (1994) Patterning C. elegans: homeotic cluster genes, cell fates and cell migrations. Trends Genet 10:159-164.

Shishido E, Takeichi M, Nose A (1998) Drosophila synapse formation: regulation by transmembrane protein with Leu-rich repeats, CAPRICIOUS. Science 280:2118-2121.

Sink H, Whitington PM (1991) Early ablation of target muscles modulates the arborisation pattern of an identified embryonic Drosophila motor axon. Development 113:701-707.

Song HJ, Ming GL, Poo MM (1997) cAMP-induced switching in turningdirection of nerve growth cones. Nature 388:275-279.
Tanabe Y, Jessell TM (1996) Diversity and pattern in the developing spinal cord. Science 274:1115-1122.

Tessier-Lavigne M, Goodman CS (1996) The molecular biology of axon guidance. Science 274:1123-1133.

Van Vactor D (1998) Protein tyrosine phosphatases in the developing nervous system. Curr Opin Cell Biol 10:174-181.

Van Vactor D, Sink H, Fambrough D, Tsoo R, Goodman CS (1993) Genes that control neuromuscular specificity in Drosophila. Cell 73:1137-1153.

Wassenberg DRD, Kronert WA, O’Donnell PT, Bernstein SI (1987) Analysis of the $5^{\prime}$ end of the Drosophila muscle myosin heavy chain gene. Alternatively spliced transcripts initiate at a single site and intron locations are conserved compared to myosin genes of other organisms. J Biol Chem 262:10741-10747.

Winberg ML, Mitchell KJ, Goodman CS (1998) Genetic analysis of the mechanisms controlling target selection: complementary and combinatorial functions of netrins, semaphorins, and IgCAMs. Cell 93:581591. 